\title{
Observation of Log-Periodic Oscillations in the Quantum Dynamics of Electrons on the One-Dimensional Fibonacci

\author{
Quasicrystal
}

\author{
Ron Lifshitz and Shahar Even-Dar Mandel \\ Raymond and Beverly Sackler School of Physics and Astronomy \\ Tel Aviv University, Tel Aviv 69978, Israel
}

June 3, 2010

\begin{abstract}
We revisit the question of quantum dynamics of electrons on the off-diagonal Fibonacci tight-binding model. We find that typical dynamical quantities, such as the probability of an electron to remain in its original position as a function of time, display log-periodic oscillations on top of the leading-order power-law decay. These periodic oscillations with the logarithm of time are similar to the oscillations that are known to exist with the logarithm of temperature in the specific heat of Fibonacci electrons, yet they offer new possibilities for the experimental observation of this unique phenomenon.
\end{abstract}

\section{Fibonacci Electrons}

The Fibonacci sequence of Long $(L)$ and Short $(S)$ intervals on the 1-dimensional line - generated by the simple substitution rules $L \rightarrow L S$ and $S \rightarrow L$-is a favorite textbook model for demonstrating the peculiar nature of electrons in quasicrystals [1-3]. The wave functions of Fibonacci electrons are neither extended nor exponentially-localized, but rather decay algebraically; the spectrum of energies is neither absolutely-continuous nor discrete, but rather singular-continuous, like a Cantor set; and the quantum dynamics is anomalous. In recent years we have studied how these three electronic properties change as the dimension of the Fibonacci quasicrystal increases to two and three [4] 8 , by constructing square and cubic versions of the Fibonacci quasicrystal [9].

The 1-dimensional off-diagonal Fibonacci tight-binding model is constructed by associating a unit hopping amplitude between sites connected by a Long interval, and a hopping amplitude $T>1$ between sites connected by a Short interval, while assuming equal on-site energies that are taken to be zero. The resulting tight-binding Schrödinger equation, on an $F_{N}$-site model, is 
given by

$$
T_{j+1} \psi(j+1)+T_{j} \psi(j-1)=E \psi(j), \quad j=1 \ldots F_{N},
$$

where $\psi(j)$ is the value of an electronic eigenfunction on site $j, E$ is the corresponding eigenvalue, $F_{N}$ is the $N^{\text {th }}$ Fibonacci number, and the hopping amplitudes $T_{j}$ are equal to 1 or $T$ according to the Fibonacci sequence $\left\{T_{j}\right\}=\{1, T, 1,1, T, 1, T, 1,1, T, 1,1, T, 1, T, 1,1, T, 1, T, \ldots\}$, as described above. The diagonal Fibonacci tight-binding model is constructed by taking all the hopping amplitudes to be equal to 1 , and taking the on-site energies to have two values, $V_{L}$ and $V_{S}$, arranged according to the Fibonacci sequence. These models have been studied extensively ever since the initial interest in the behavior of electrons in quasiperiodic potentials [10 13, and continue to offer mathematical challenges to this day [14].

Various 2-dimensional extensions of the Fibonacci model were introduced soon thereafter [15[19], and strongly promoted recently [9] as models for quasicrystals without 'forbidden' symmetries [20,21]. In our studies, we have shown that whereas Fibonacci electrons in one dimension always behave as described above for any $T>1$, in two dimensions, and even more so in three, there is crossover - as the strength $T$ of the quasiperiodicity approaches 1 - to a regime in which Fibonacci electrons behave more and more like electrons do in periodic crystals, particularly in the sense that their energy spectra develop continuous intervals [46]. These results were recently explained in a rigorous manner by Damanik and Gorodetzki [22]. More surprisingly, our studies of Fibonacci electrons have led us to new results also in the simple 1-dimensional case. We have examined dynamical properties, such as the probability of an electron to remain in its original position as a function of time. The power-law decay of this quantity is commonly used for analyzing the dynamics. Surprisingly, we have observed log-periodic oscillations on top of the power-law decay, implying the existence of an imaginary correction to the exponent. We wish to describe this new observation here.

\section{Quantum Dynamics of Electronic Wave-Packets}

We consider the dynamics of electronic wave-packets, or states $|n\rangle$, that are initially localized at a single lattice site $n$ of the 1-dimensional Fibonacci quasicrystal, denoting their amplitude on site $m$ at time $t$ by $\phi_{n}(m, t)$. Thus, at time $t=0$ the wave-packet is given by $\phi_{n}(m, 0)=\langle m \mid n\rangle=\delta_{m n}$, where $\delta_{m n}$ is the Kronecker delta. At any later time $t>0$ the wave-packet is given by $e^{i H t}|n\rangle$, which is simply the $n^{\text {th }}$ column of the matrix representation of the time evolution operator $e^{i H t}$, where $H$ is the off-diagonal matrix representation of the Hamiltonian in Eq. (1), and we take $\hbar=1$. Thus, $\phi_{n}(m, t)=\left\langle m\left|e^{i H t}\right| n\right\rangle=\left(e^{i H t}\right)_{m n}$.

We characterize the dynamics of wave packets by monitoring two typical quantities: (a) The Survival Probability of the $n^{\text {th }}$ wave-packet, defined as the probability of finding the electron at 
its initial position at time $t$, is given by

$$
S_{n}(t)=\left|\phi_{n}(n, t)\right|^{2}=\left|\left(e^{i H t}\right)_{n n}\right|^{2} ;
$$

and (b) The Inverse Participation Ratio of the $n^{\text {th }}$ wave-packet, which measures the spatial extent of the wave-packet, is given by

$$
I_{n}(t)=\frac{\sum_{m}\left|\phi_{n}(m, t)\right|^{4}}{\left(\sum_{m}\left|\phi_{n}(m, t)\right|^{2}\right)^{2}}=\sum_{m}\left|\left(e^{i H t}\right)_{m n}\right|^{4}
$$

where the last equality holds because the wave-packets are normalized. Both of these quantities are often used to examine the dynamics of wave-packets. In particular, the manner in which they decay as the wave-packets spread with time is associated with different regimes of the quantum dynamics. The actual calculation is performed numerically by applying the discrete time-evolution operator $e^{i H \Delta t}$ successively to the initial conditions. The time-step $\Delta t$ is kept sufficiently small to satisfy the Nyquist criterion by noting that the largest eigenvalue of $H$ is bounded by $1+T$, thus requiring $\Delta t$ to be smaller than $1 /(2+2 T)$.

If a function $F(t)$ decays asymptotically with some power law $F(t) \sim t^{-\beta}$, then the exponent $\beta$ can be found by

$$
\beta=\lim _{t \rightarrow \infty}-\frac{\ln F(t)}{\ln t},
$$

but in general the exponent $\beta$ is not guaranteed to exist. The bounds $\beta_{ \pm}$on $\beta$, which always exist, are given by

$$
\beta_{+}=-\limsup _{t \rightarrow \infty} \frac{\ln F(t)}{\ln t} ; \quad \beta_{-}=-\liminf _{t \rightarrow \infty} \frac{\ln F(t)}{\ln t} ;
$$

and if $\beta_{+}=\beta_{-}$then $\beta$ exists. Furthermore, if an exponent $0<\beta<1$ characterizes the power-law decay of a function $F(t)$, then the same exponent also characterizes the decay of the time-averaged function

$$
\langle F\rangle_{t}=\frac{1}{t} \int_{0}^{t} F\left(t^{\prime}\right) \mathrm{d} t^{\prime}
$$

We use the definition of Eq. (5) to study the long-time asymptotic values of the exponents $\beta_{S}$ related to the survival probability of a wave-packet and $\beta_{I}$ related to the inverse participation ratio of the wave-packet. In what follows we are mostly concerned with the early-time behavior of these exponents.

Damanik et al. [14,23] recently used the second moment of the position operator for the diagonal tight-binding Hamiltonian, to show that far from the periodic limit, or very close to it, the dynamics of wave-packets is independent of the initial site. However, since we are interested in intermediate values of $T$ as well - equivalent in the diagonal model to intermediate values of the difference $\left|V_{L}-V_{S}\right|$ between the different on-site energies - we expect to find different dynamical behavior depending on the choice of the initial site. Thus, we typically examine the maximal, 


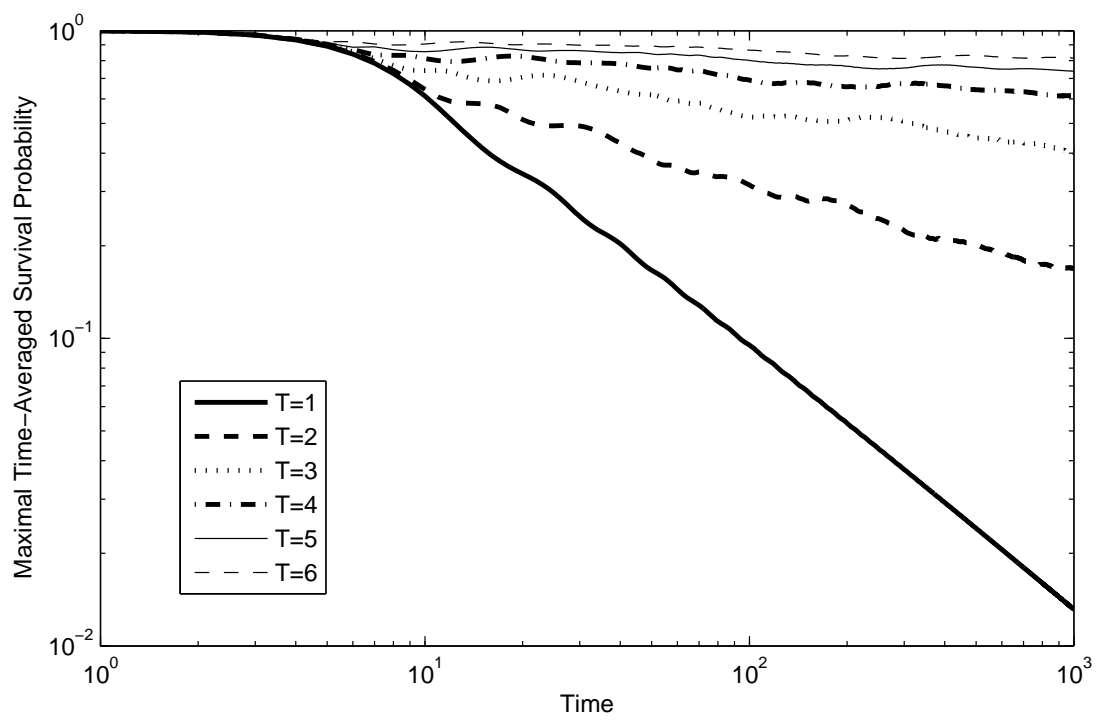

Figure 1: The time-averaged maximal survival probability for a 233 -site $1 d$ approximant, with periodic boundary conditions, calculated for different values of $T$. The asymptotic behavior of the slopes allows to extract the diffusion exponent $\beta_{S}^{\max }$ as a function of $T$, which is displayed in Fig. 2 ,

the minimal, and the site-averaged survival probabilities and inverse participation ratios, all of which display similar qualitative behavior. In Ref. [8] we study the different exponents in detail, in one, two, and three dimensions. Here we concentrate on the $1 d$ results for the maximal survival probability exponent $\beta_{S}^{\max }$.

\section{Asymptotic Behavior of the Maximal Survival Probability}

We look at the wave-packet whose time-averaged survival probability is maximal. Fig. 1 shows the time-averaged maximal survival probability $\left\langle S_{\max }\right\rangle_{t}$ on a log-log scale for a 233 -site $1 d$ approximant, with periodic boundary conditions, for different values of $T$. The exponents extracted from the slopes of the curves in Fig. 1 for four orders of approximants are shown in Fig. 2, as functions of $T$.

Convergence of the exponent $\beta_{S}^{\max }$ is evident for values of $T>2$ even for relatively small approximants. Convergence is not obtained for smaller values of $T$ that approach the periodic limit $T \rightarrow 1$, where the dynamics is expected to become ballistic. In this limit the wavepackets quickly spread out and the finite size of the approximant has a stronger influence on the dynamics. Despite this limitation, detailed studies of the slopes, for increasing orders of approximants and for varying time-ranges clearly show that the extracted exponents converge, as the order of the approximant increases, and longer averaging times are possible. We note 


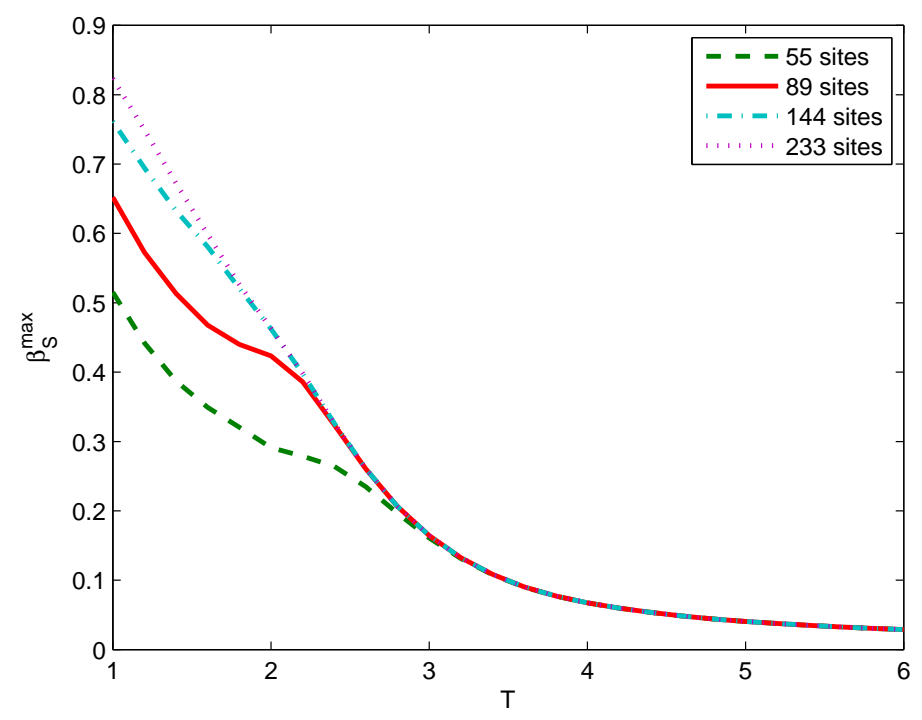

Figure 2: The exponent $\beta_{S}^{\max }$ as a function of $T$ for increasing orders of approximants. Convergence is improved for decreasing values of $T$ as the order of the approximant is increased.

that Similar curves are obtained for the minimal and the site-averaged survival probabilities, as well as for the minimal, maximal, and site-averaged inverse participation ratios [8].

\section{Log-Periodic Oscillations and the Fourier Transform of the Density of States}

A closer inspection of the temporal decay of the survival probabilities in Fig. 1 reveals a small oscillating behavior on top of the overall leading power-law. In order to better expose this behavior, we divide out the leading asymptotic power-law $t^{-\beta}$ that was found earlier, and obtain the curves shown in Fig. 3. Similar results are observed in the study of the inverse participation ratio. The curves clearly exhibit log-periodic oscillations - oscillation that are periodic in $\log (t)$-around the mean value of 1 , especially for the larger values of $T$. These oscillations contain a basic frequency $\omega$ that seems to decrease with increasing $T$, as well as a sequence of higher-frequency oscillations with decreasing amplitudes that seem to develop with time. The fundamental oscillations can be described empirically by a temporal decay with a complex exponent of the form

$$
f(t) \propto t^{-\beta}+\alpha t^{-\beta \pm i \omega}+(\text { corrections } \ll \alpha),
$$

which after dividing out the leading power-law term become

$$
\frac{f(t)}{t^{-\beta}} \propto 1+\alpha t^{ \pm i \omega}=1+\alpha e^{ \pm i \omega \log (t)}
$$



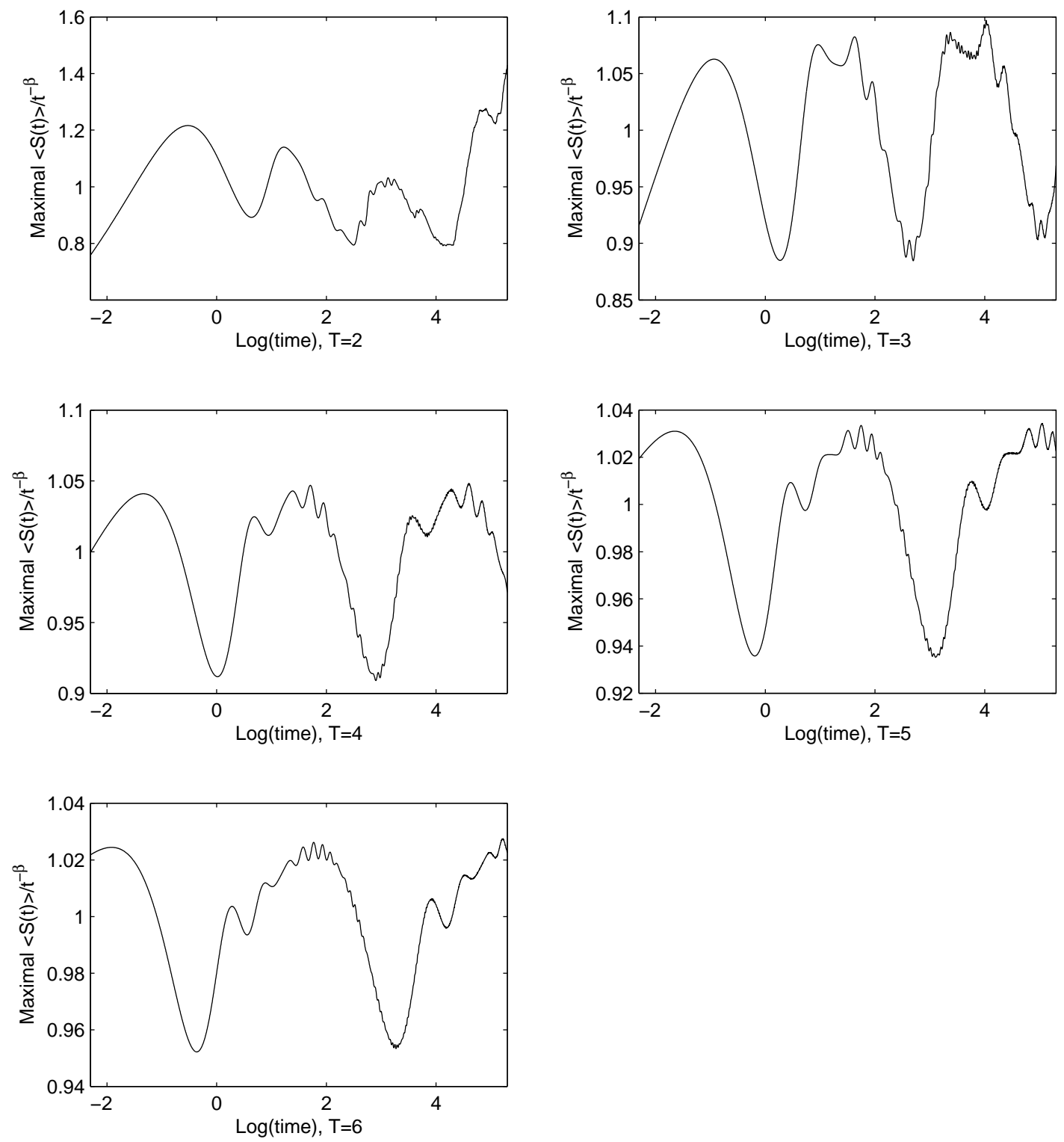

Figure 3: Log-periodic oscillations in the maximal survival probability, after dividing out the leading power-law decay, for an 89-site approximant with 5 different values of $T$. In addition to the basic periodic oscillation one observes the emergence of higher-frequency oscillations as time advances. 
yielding oscillations of amplitude $\alpha$ around 1 that are periodic in $\log (t)$ with frequency $\omega$.

Log-periodic oscillations are not as uncommon in physics as one may think. They appear in critical phenomena 24 26], where renormalization-group calculations yield power-law behavior with complex exponents near phase transitions; they also appear quite generally in problems related to random-walks or classical diffusion on self-similar systems or fractals, where the fractal dimension turns out to be complex [27-31]. As such, they naturally occur in quasiperiodic systems like the Fibonacci quasicrystal, where the spectrum of energies is multifractal. This has been observed in quasiperiodic Ising models [32, and in specific heat studies of the tight-binding Fibonacci model [33 37].

In specific heat studies, where log-periodic oscillations appear in temperature, the object of calculation is the partition function

$$
Z\left(\beta_{\mathrm{T}}\right)=\sum_{k} e^{-\beta_{\mathrm{T}} E_{k}}
$$

where $\beta_{\mathrm{T}}$ is the inverse temperature; whereas in problems of diffusion [31], the object of calculation is the so-called heat kernel of the diffusion equation

$$
Z(t)=\sum_{k} e^{-E_{k} t}
$$

In both cases, when expressed in integral form, these are Laplace transforms of the density of states. To see the relation to the present problem of quantum dynamics, we expand the expression for the survival probability (2) in eigenstates of the Hamiltonian,

$$
S_{n}(t)=\left|\sum_{k}\left\langle n\left|e^{i H t}\right| \psi_{k}\right\rangle\left\langle\psi_{k} \mid n\right\rangle\right|^{2}=\left.\left.\left|\sum_{k}\right| \psi_{k}(n)\right|^{2} e^{i E_{k} t}\right|^{2} .
$$

Thus, what we are calculating is the magnitude-squared of the Fourier transform of the density of states, weighted by the overlap of each eigenstate with the initial state $|n\rangle$.

Equation (11) is easily calculated in the periodic limit $T \rightarrow 1$ of our model, where the eigenstates are Bloch function, with $\left|\psi_{k}(n)\right|^{2}=1 / N$ independent of $n$, and the eigenvalues form a single band with dispersion $E_{k}=\cos (k)$. This yields

$$
S_{T=1}(t) \propto\left|\int_{-\pi}^{\pi} \mathrm{d} k e^{i t \cos k}\right|^{2} \propto J_{0}^{2}(t)
$$

where $J_{0}(t)$ is the zeroth order Bessel function of the first kind, which is known to decay asymptotically as $t^{-1 / 2}$. This is consistent with the expected ballistic dynamics of Bloch electrons in a periodic crystal. The time average of Eq. (12) is approximately the behavior calculated numerically for $T=1$ on a 233-site approximant, and shown in Fig. 1, Note that the fine wiggles that are barely seen at very early times are associated with the first few zeros of the Bessel function, 
and are not log-periodic oscillations. Log-periodic oscillations appear for $T>1$ as a result of the multifractal nature of the Fibonacci spectrum, for similar reasons as in the calculation of the partition function (9) [36, 37]. A more detailed analysis of Eq. (11) for $T>1$ is required to quantitatively characterize the spectral properties of the log-periodic oscillations, which we have discovered here by numerical means.

Log-periodic oscillations in the specific heat of Fibonacci quasicrystals near zero temperature are very difficult to observe experimentally. The discovery of log-periodic oscillations in the quantum dynamics of wave-packets in Fibonacci quasicrystals should open new possibilities for the actual experimental observation of this unique phenomenon. A particular realization could be in optical experiments that allow one to observe the dynamics of wave-packets within 1dimensional [38], as well as 2-dimensional [39], photonic quasicrystals. Thus, we hope that our numerical observations here will stimulate further studies, analytical and experimental alike.

\section{Acknowledgments}

We wish to thank Amnon Aharony for fruitful discussions and for suggesting that the "wiggles" in our numerical data might be real. We also thank Eric Akkermans and Eli Eisenberg for helpful discussions. This research is supported by the Israel Science Foundation through Grant No. $684 / 06$.

\section{References}

[1] T. Janssen. In R.V. Moody, editor, The Mathematics of Long-Range Aperiodic Order, page 269. Kluwer, Dordrecht, 1997.

[2] T. Fujiwara. Theory of electronic structure in quasicrystals. In Z.M. Stadnik, editor, Physical Properties of Quasicrystals, chapter 6. Springer, Berlin, 1999.

[3] David Damanik. Gordon-type arguments in the spectral theory of one-dimensional quasicrystals. In M. Baake and R.V. Moody, editors, Direction in Mathematical Quasicrystals, pages 277-304. AMS, Providence, 2000.

[4] R. Ilan, E. Liberty, S. Even-Dar Mandel, and R. Lifshitz. Electrons and phonons on the square Fibonacci tilings. Ferroelectrics, 305:15, 2004.

[5] Shahar Even-Dar Mandel and Ron Lifshitz. Electronic energy spectra and wave functions on the square Fibonacci tiling. Phil. Mag., 86:759, 2006.

[6] Shahar Even-Dar Mandel and Ron Lifshitz. Electronic energy spectra of square and cubic Fibonacci quasicrystals. Phil. Mag., 88:2261, 2008.

[7] S. Even-Dar Mandel and R. Lifshitz. Bloch-like electronic wave functions in two-dimensional quasicrystals. Preprint (arXiv:0808.3659).

[8] S. Even-Dar Mandel and R. Lifshitz. Quantum dynamics of electronic wave-packets on Fibonacci quasicrystals. In preparation.

[9] R. Lifshitz. The square Fibonacci tiling. J. of Alloys and Compounds, 342:186-190, 2002.

[10] M. Kohmoto, L. P. Kadanoff, and C. Tang. Localization problem in one dimension: Mapping and escape. Phys. Rev. Lett., 50:1870-1872, 1983. 
[11] S. Ostlund, R. Pandit, D. Rand, H. S. Schellnhuber, and E. D. Siggia. One-dimensional Schrödinger equation with an almost periodic potential. Phys. Rev. Lett., 50:1873-1876, 1983.

[12] M. Kohmoto and J.R. Banavar. Quasiperiodic lattice: Electronic properties, phonon properties, and diffusion. Phys. Rev. B, 34:563-566, 1986.

[13] M. Kohmoto, B. Sutherland, and C. Tang. Critical wave functions and a Cantor-set spectrum of a one-dimensional quasicrystal model. Phys. Rev. B, 35:1020-1033, 1987.

[14] David Damanik, Mark Embree, Anton Gorodetski, and Serguei Tcheremchantsev. The fractal dimension of the spectrum of the Fibonacci hamiltonian. Commun. Math. Phys., 280:499-516, 2008.

[15] K. Ueda and H. Tsunetsungu. Energy spectrum and conductance of a two-dimensional quasicrystal. Phys. Rev. Lett., 58:1272-1275, 1987.

[16] W.A. Schwalm and M.K. Schwalm. Extension theory for lattice Green functions. Phys. Rev. B, 37:9524, 1988.

[17] J.X. Zhong and R. Mosseri. Quantum dynamics in quasiperiodic systems. J. Phys: Condens. Matter, 7:8383, 1995.

[18] S. Roche and D. Mayou. Conductivity of quasiperiodic systems: A numerical study. Phys. Rev. Lett., 79:2518, 1997.

[19] Yu.Kh. Vekilov, I.A. Gordeev, and E.I. Isaev. Electronic spectrum of a two-dimensional Fibonacci lattice. JETP, 89:995, 1999.

[20] R. Lifshitz. What is a crystal? Z. Kristallogr., 222:313, 2007.

[21] R. Lifshitz. Quasicrystals: A matter of definition. Foundations of Physics, 33:1703, 2003.

[22] David Damanik and Anton Gorodetski. The spectrum of the weakly coupled Fibonacci hamiltonian. Electron. Res. Announc. Amer. Math. Soc., 16:23-29, 2009.

[23] David Damanik, Rowan Killip, and Barry Simon. Perturbations of orthogonal polynomials with periodic recursion coefficients. Ann. of Math., 171:1931-2010, 2010.

[24] M. Nauenberg. Scaling representation for critical phenomena. J Phys. A: Math. Gen., 8:925, 1975.

[25] Th. Niemeijer and J. van Leeuwen. In C. Domb and M. S. Green, editors, Phase Transitions and Critical Phenomena, volume 6. Academic Press, New York, 1976.

[26] R. Jullien, K. Uzelac, P. Pfeuty, and P. Moussa. The Yang-Lee edge singularity studied by a four-level quantum renormalization-group blocking method. J. Phys. France, 42:1075, 1981.

[27] Barry D. Hughes, Michael F. Shlesinger, and Elliott W. Montroll. Random walks with self-similar clusters. Proc. Natl. Acad. Sci. USA, 78:3287-3291, 1981.

[28] Robert B. Griffiths and Miron Kaufman. Spin systems on hierarchical lattices. Introduction and thermodynamic limit. Phys. Rev. B, 26:5022, 1982.

[29] B. Derrida, C. Itzykson, and J. M. Luck. Oscillatory critical amplitudes in hierarchical models. Commun. Math. Phys., 94:115-132, 1984.

[30] D. Sornette, A. Johansen, A. Arneodo, J. F. Muzy, and H. Saleur. Complex fractal dimensions describe the hierarchical structure of diffusion-limited-aggregate clusters. Phys. Rev. Lett., 76:251$254,1996$.

[31] E. Akkermans, G. V. Dunne, and A. Teplyaev. Physical consequences of complex dimensions of fractals. Europhys. Lett., 88:40007, 2009.

[32] R. F. S. Andrade. Detailed characterization of log-periodic oscillations for an aperiodic Ising model. Phys. Rev. E, 61:7196-7199, 2000. 
[33] Raúl O. Vallejos, Renio S. Mendes, Luciano R. da Silva, and Constantino Tsallis. Connection between energy spectrum, self-similarity, and specific heat log-periodicity. Phys. Rev. E, 58:1346-1351, 1998.

[34] L. R. da Silva, R. O. Vallejos, C. Tsallis, R. S. Mendes, and S. Roux. Specific heat of multifractal energy spectra. Phys. Rev. E, 64:011104, 2001.

[35] I N de Oliveira, M L Lyra, E L Albuquerque, and L R da Silva. Bosons with multifractal energy spectrum: Specific heat log periodicity and Bose-Einstein condensation. J. Phys.: Condens. Matter, $17: 3499,2005$.

[36] P. W. Mauriz, E. L. Albuquerque, and M. S. Vasconcelos. Electronic specific heat properties in one-dimensional quasicrystals. Physica A, 294:403-414, 2001.

[37] P. W. Mauriz, M. S. Vasconcelos, and E. L. Albuquerque. Specific heat properties of electrons in generalized Fibonacci quasicrystals. Physica A, 329:101-113, 2003.

[38] Y. Lahini, R. Pugatch, F. Pozzi, M. Sorel, R. Morandotti, N. Davidson, and Y. Silberberg. Observation of a localization transition in quasiperiodic photonic lattices. Phys. Rev. Lett., 103:013901, 2009.

[39] Barak Freedman, Guy Bartal, Mordechai Segev, Ron Lifshitz, Demetrios N. Christodoulides, and Jason W. Fleischer. Observation of wave and defect dynamics in nonlinear photonic quasicrystals. Nature, 440:1166-1169, 2006. 